\title{
An event-related fMRI study of the neural networks underlying repetition suppression and reaction time priming in implicit visual memory
}

\author{
Christian Habeck ${ }^{a, *}$, H. John Hilton ${ }^{a, b}$, Eric Zarahn ${ }^{a, c}$, \\ Truman Brown ${ }^{d}$, Yaakov Stern ${ }^{a, b, c, e}$
}

${ }^{a}$ Cognitive Neuroscience Division of the Taub Institute for Research in Alzheimer's Disease and the Aging Brain, 622 West 168th Street, PH-18, New York, NY 10039, USA

${ }^{\mathrm{b}}$ Department of Neurology, College of Physicians and Surgeons of Columbia University, 630 West 168th Street, New York, NY 10027, USA

${ }^{c}$ Department of Psychiatry, College of Physicians and Surgeons of Columbia University, 630 West 168th Street, New York, NY 10027, USA

${ }^{\mathrm{d} D e p a r t m e n t}$ of Biomedical Engineering, College of Physicians and Surgeons of Columbia University, 630 West 168th Street, New York, NY 10027, USA

${ }^{\mathrm{e}}$ Department of Psychology, College of Physicians and Surgeons of Columbia University, 630 West 168th Street, New York, NY 10027, USA

\section{A R T I C L E I N F O}

Article history:

Accepted 23 November 2005

Available online 14 February 2006

Keywords:

Covariance analysis

Functional imaging

Neural network

Visual recognition memory

\begin{abstract}
A B S T R A C T
Unfamiliar line drawings were presented to subjects three times during BOLD fMRI scanning. A set of brain areas was detected in which the effect of stimulus repetition on the evoked fMRI response depended on whether or not the drawing could be conceived as a coherent three-dimensional structure. Differential repetition effects were found in the neural response to drawings of both structurally possible and impossible objects. This differential effect of repetition was related to the amount of reaction time priming on the concurrent task involving decisions about three-dimensional structure in the possible but not in the impossible objects. These results point to different neurophysiological processing mechanisms for structurally possible and impossible images and demonstrate neural plasticity that predicts behavioral priming for structurally possible images.
\end{abstract}

() 2005 Elsevier B.V. All rights reserved.

\section{Introduction}

In a PET activation study of implicit and explicit memory, Schacter et al. (1995) found object-dependent activation effects. In particular, they observed increased CBF in the area of the left temporal and fusiform gyri when subjects made possible/impossible object decisions on previously encoded possible but not impossible objects. No such trends were observed for new objects. This finding was used as support for the role of a Structural Description System (SDS) that computes three-dimensional structural descriptions of visual events. As impossible objects lack a globally coherent three-dimensional geometry, the SDS is unable to successfully compute a singular three-dimensional representation of an impossible object.

While highly compelling, this study fails to address two fundamental concerns. First, it was done using PET imaging in a blocked design. Although attributable to the conditions of state-of-the-art neuroimaging at the time, this is a serious methodological flaw that in and of itself justifies repeating the experiment. Furthermore, as a blocked design, all trials within

\footnotetext{
* Corresponding author. 630 West 168th Street, P\&S Box 16, New York, NY 10032, USA. Fax: +1 2123421838.

E-mail address: ch629@columbia.edu (C. Habeck).
} 
a given block have primarily the same set of experimental conditions (e.g., possible, encoded). Blocked designs in memory experiments are thus very undesirable since they do not establish a rigorously controlled randomized task structure and thus cannot guarantee the elimination of confounding influences that could enable the subjects to by-pass the actual object decision itself (and thus respond, after the first few trials, simply on the basis of the block structure and not stimulus type). Second, there was no behavioral priming effect on the possible/impossible object decision for the impossible objects. This makes it difficult to establish that the observed specificity of the neural activations is due to object type per se and not due to whether other object or task factors may have reduced priming specifically for the impossible objects.

Any investigation of the neural networks underlying cognitive activity must consider the nature of the neural signals involved. It has been proposed that neural response, and by extension hemodynamic repetition suppression (Desimone, 1996; Wiggs and Martin, 1998), are at the basis of perceptual priming mechanisms. Suppression as well as latency reduction of neural responses (as measured by EEG) is thought to occur with the lowering of the perceptual threshold for stimulus identification after repeated exposures (Wiggs and Martin, 1998), which does not require any explicit recollection of having seen the object before.

Despite the evidence linking repetition suppression to perceptual priming across a variety of object decision and identification tasks, the assertion of a linkage between the two phenomena has recently come under criticism in a series of articles (Henson and Rugg, 2003; Henson et al., 2002; Eger et al., 2004) that bring up important questions. Henson and Rugg (2003) systematically scrutinize all of the steps in the link between repetition suppression and perceptual priming formulated in Wiggs and Martin (1998). This link partially relies on implicit assumptions about (1) the mapping between neural activity and hemodynamic response, (2) the mapping between hemodynamic response and behavior, and (3) the mapping between neural activity and behavior.

With regard to the mapping between neural activity and hemodynamic response, Henson and Rugg argue that the generic phenomenon of neural response suppression observed in animals when exposed to repeated stimuli (Brown and Xiang, 1998) might happen too fast to be compatible with the peak and latency reductions observed in human ERP and fMRI studies. Rather, the integrative nature of signal modalities in human neuroimaging studies of higher cognition might reflect modulation by, and interaction with, other brain areas. For example, repetition effects commonly found in the lateral occipital complex (Grill-Spector et al., 1999) might not be directly attributable to response suppression of neurons residing at that brain location. Further, Henson and Rugg note in light of residual uncertainty in the biophysical relationship between neural activity and hemodynamic response (Logothetis et al., 2001) that even if there was a direct link between neural and hemodynamic responses at particular brain locations, the sign of the relationship would not be unequivocally clear. We have tried to accommodate these concerns and chosen a multivariate approach in our data analysis; this approach pays attention to interactions between brain areas and changes in activation across stimulus repetitions of both signs.

Henson and Rugg (2003) note further that the presumed relationship between hemodynamic/neural response suppression and perceptual learning can show dissociation in certain circumstances: for instance, repetition effects are usually not found in early sensory or motor regions (Schacter and Buckner, 1998) although it is equally plausible for these regions to be modulated by attention or reaction time as the more commonly found lateral occipital complex. Additionally, as shown in a recent report (Dobbins et al., 2004), the type of perceptual learning influences whether response suppression is present. In the cited report, priming was found with concomitant cortical deactivations across repeated presentations for a fixed relational object-size judgment ("bigger/ smaller than a shoebox"). However, switching the relational size judgment to its logical complement ("bigger" $\rightarrow$ "smaller") between repetitions abolishes most priming behavior and cortical de-activation, hinting at the learning of the stimulusresponse association, rather than a true facilitation of objectrelated knowledge. Other studies have also found that hemodynamic response suppression requires that objects are attended to (Eger et al., 2004), challenging the automaticity of neural response suppression for repeated exposures to visual stimuli which was also suggested by Wiggs and Martin (1998).

In the current study, we set out to probe the link between priming and repetition effects in a modified trial-based version of the possible/impossible object decision (PI-OD) task (Schacter and Cooper, 1993, 1995) using event-related fMRI. Using efMRI and a modified trial-based PI-OD paradigm resolves the two concerns stemming from the original Schacter and Cooper PET findings. By contrast to the trialbased paradigm, the PI-OD task has typically been implemented as a block-mediated priming paradigm consisting of an initial structural encoding phase, where subjects view both types of objects without explicit awareness of the structural possibility attribute, followed by the object decision test block. In the object decision block, subjects indicate whether intermixed encoded and new possible and impossible stimuli are indeed "impossible" or "possible" in structure. Sample possible and impossible objects are shown in Fig. 1.

This design has revealed priming effects for possible, but not for impossible objects, and involved the left inferior temporal gyrus (Schacter et al., 1995). Our trial-mediated
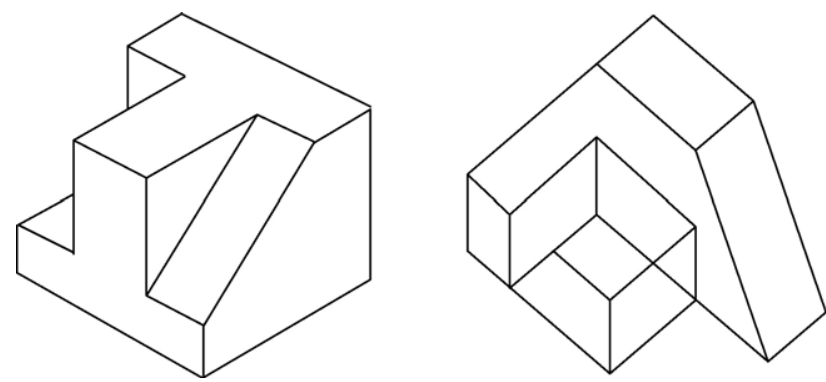

Fig. 1 - Examples of possible (P) and impossible (I) objects used in the object decision task. Left: P-object; right: I-object. 
modification shortens the delay between prime and target from approximately $10 \mathrm{~min}$ to an order of seconds, maintains the same task for prime and target, and allows the measurement of incremental priming effects across a series of stimulus presentations. These manipulations were intended to increase our power for detecting behavioral priming and its neural underpinnings in a smaller group of subjects. Our multivariate analysis (explained in the Experimental procedure section) takes advantage of this repeated measures design to pinpoint neural activation patterns that show reliable within-subjects repetition effects for as many subject as possible. This is a more stringent requirement than the more liberal rule of mean subject changes in activation across repetitions.

Researchers have primarily focused on the lateral occipital complex and its role in object perception and perceptual priming (Kourtzi and Kanwisher, 2000, 2001). We decided to perform a brain-wide search for repetition effects and employed a multivariate analysis technique to find regionally correlated changes in response to repeated presentation of items. If such changes are present, a multivariate analysis has a higher chance of detecting them than a voxel-wise univariate technique, without restriction to a particular brain region. Our technique would ideally pinpoint the lateral occipital cortex as part of a network of brain regions that modulates its activity in response to repeated item presentations and show how this might be related to perceptual priming.

Fig. 2 gives a schematic sketch of two different scenarios. We stress that the figure contains no actual data-only hypothetical data are used for three regions of interest: the lateral occipital cortex, a prefrontal region, and a parietal region. One scan for each subject and each condition can therefore be represented as a point in a three-dimensional space. The figure shows simulated data from 15 subjects over three stimulus presentations. Different repetitions are color coded: yellow denotes presentation 1, red denotes presentation 2, and blue denotes presentation 3. The sequence of scans for a representative subject is indicated with the numerals ' 1 ', ' 2 ', and ' 3 '. The sketch on the right shows the scenario best suited for our analytic method (explained in the Data analysis section). All three regions of interest decrease their activation in concert across the sequence of presentations. The thin blue line denotes the covariance pattern as a vector indicating a fixed direction in the three-dimensional region-of-interest space. In this example, this vector has the regional weight values $[1 ; 1$; 1], implying equal amounts of change in activation in all three regions of interest. [Again, this is just our convenient choice for an educational example; in general, regional weights in covariance patterns show varying magnitudes and signs across regions.] Repetition suppression can be visualized as moving along this line towards the origin of the three-dimensional coordinate system. The left part of the figure shows a scenario unsuited for multivariate analysis and more appropriate for univariate analysis such as SPM. Here, there is no correlation in activations across the regions of interest. The lateral occipital cortex still shows a repetition suppression effect, but it has no consistent relationship with the other two regions of interest. The sequence of Arabic numerals from 1 to 3 now does not move
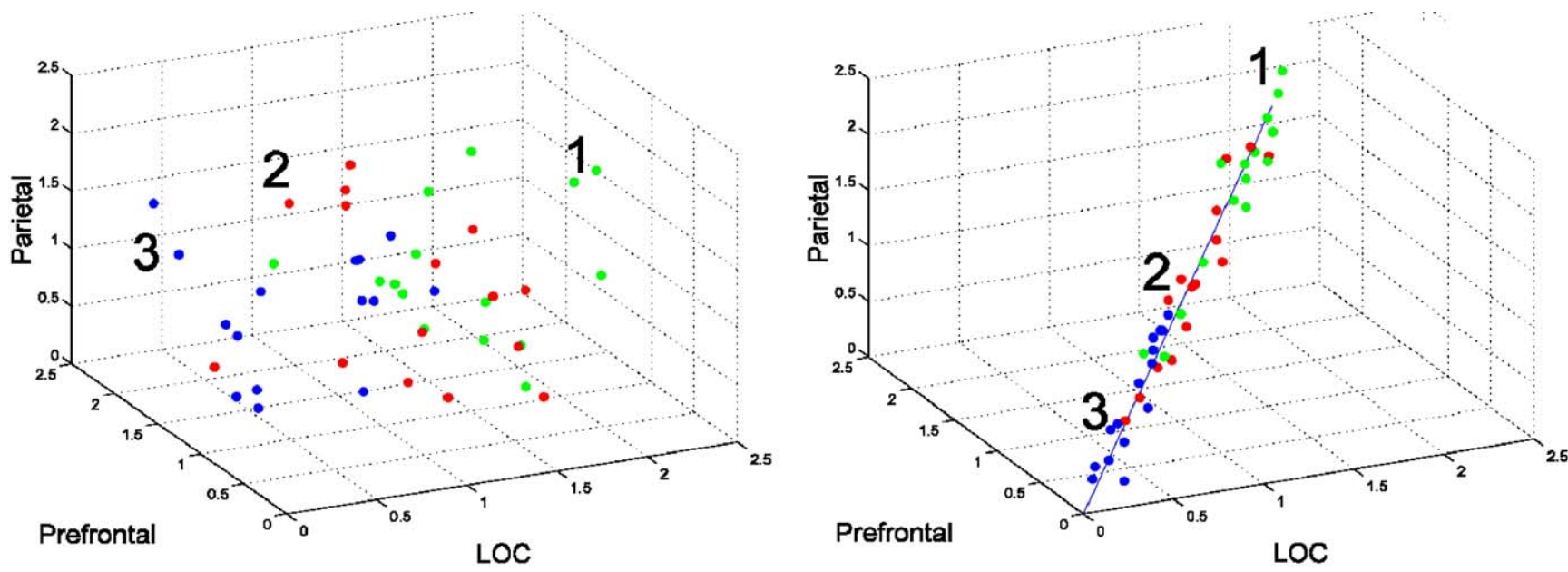

Fig. 2 - A mock example of simulated data explaining the difference between univariate and multivariate analysis: the figure shows 15 subjects, scanned three times repeatedly. Different repetitions are color coded: yellow denotes presentation 1, red denotes presentation 2, and blue denotes presentation 3. The regions of interest picked are indicated as axis labels: the lateral occipital complex (LOC) denotes the $x$-axis, a pre-frontal region denotes the $y$-axis, and a parietal region denotes the $z$-axis. The sequence of scans for one subject only is indicated with the Arabic numerals ' 1 ', ' 2 ', and ' 3 '. Right: the graph on the right shows a regionally correlated repetition effect. All three regions of interest decrease their activation in concert across the sequence of presentations. The thin blue line denotes the covariance pattern as a vector indicating a fixed direction in the threedimensional region-of-interest space. Repetition suppression can be visualized as moving along this line towards the origin of the three-dimensional coordinate system. Left: the left part of the figure shows a scenario with no regional correlation between the regions of interest. The LOC does still show a repetition suppression effect, but it has no consistent relationship with the other two regions of interest. The sequence of Arabic numerals from 1 to 3 now does not move along one line any longer and only displays a correct ordering when projected onto the LOC axis. 
along a single line and only displays a correct ordering when projected onto the LOC-axis.

We can summarize the goals of this study with these guiding questions for both possible and impossible objects:

(1) Are there reliable neural repetition effects?

(2) Are there reliable priming effects?

(2) If both are present, are they related with each other?

(4) Is the lateral occipital complex part of any covariance pattern underlying repetition effects? What other areas are included, i.e., show an interaction with the lateral occipital complex?

We anticipated a positive answer to the first 3 questions for possible objects. Answers to all other questions are as yet open.

Although this study was not designed to obviate the earlier concerns about the prominent role that learning of stimulusresponse associations can play at the expense of true object decisions (Dobbins et al., 2004; Schacter et al., 2004), behavioral data from our subjects are at our disposal, allowing us to address the following question in our analysis. If perceptual learning involves the strengthening of stimulus-response associations without regard to the object decision, we would anticipate that incorrect decisions made for the first presentation of an object would persist in subsequent trials. Thus, the conditional probability of making incorrect subsequent decisions given incorrect initial decisions (i.e., persisting with the initial erroneous stimulus-response association) should be larger than the probability of reversing the stimulus-response association and making a correct decision. Also, it follows for this scenario that there should not be any effect of object presentation on the accuracy of the object decision even in the larger sample.

\section{Results}

\subsection{Behavioral performance}

We use reaction time as the main behavioral variable (see Table 1). When subjected to a repeated measures ANOVA, there was a clear effect of presentation $(F(3,39)=38.89$, $P<0.0001)$, an effect of object type $(F(1,13)=13.34, P<0.005)$, but no object type-presentation interaction $(F(3,39)=2.57$, $P=0.08$ ). (All corrected according to Huyn-Feldt epsilon criterion.)

Table 1 - Reaction times as a function of presentation and object type

\begin{tabular}{lcc} 
& Possible & Impossible \\
\hline Presentation 1 & $666 \pm 64$ & $761 \pm 154$ \\
Presentation 2 & $594 \pm 63$ & $732 \pm 158$ \\
Presentation 3 & $579 \pm 69$ & $700 \pm 127$ \\
Presentation 4 & $567 \pm 54$ & $651 \pm 100$ \\
\hline
\end{tabular}

Units are in milliseconds. There was an effect of presentation $(F$ $(3,39)=38.89, P<0.0001)$, an effect of object type $(F(1,13)=13.34$, $P<0.005)$, but no object type-presentation interaction $(F(3,39)=2.57$, $P=0.08$ ).
Table 2 - Accuracy as a function of presentation and

object type

\begin{tabular}{|c|c|c|c|c|}
\hline & \multicolumn{2}{|c|}{ Percent correct } & \multirow[t]{2}{*}{$d^{\prime}$} & \multirow[t]{2}{*}{$C_{\ln }$} \\
\hline & Possible & Impossible & & \\
\hline Presentation 1 & $0.96 \pm 0.04$ & $0.87 \pm 0.08$ & $2.92 \pm 0.56$ & $-0.66 \pm 0.57$ \\
\hline Presentation 2 & $0.94 \pm 0.07$ & $0.87 \pm 0.08$ & $2.96 \pm 0.87$ & $-0.61 \pm 0.55$ \\
\hline Presentation 3 & $0.96 \pm 0.04$ & $0.88 \pm 0.09$ & $2.97 \pm 0.79$ & $-0.49 \pm 0.34$ \\
\hline Presentation 4 & $0.96 \pm 0.06$ & $0.92 \pm 0.06$ & $3.14 \pm 0.80$ & $-0.37 \pm 0.46$ \\
\hline \multicolumn{5}{|c|}{$\begin{array}{l}\text { There was an effect of object type with higher percent correct for } \\
\text { recognition with the possible objects }(F(1,13)=36.33, P<0.0001) \text {, but } \\
\text { no effect of presentation }(F(3,39)=1.60, P=0.20) \text { and no interaction } \\
\text { between object type and presentation }(F(3,39)=2.62, P=0.06) \text {. There } \\
\text { was no effect of presentation on either } d^{\prime}(F(3,39)=0.82, P=0.49) \text { or } \\
C_{\ln }(F(3,39)=1.57, P=0.21) \text { measures of signal detection. }\end{array}$} \\
\hline
\end{tabular}

Recognition accuracy was recorded, but not used for any further analysis (see Table 2). It was computed for each item type and presentation number separately as the number of hits and correct rejections divided by the number of trials, with both numerator and denominator taken at the item type and presentation number in question. Predictably, there was an effect of object type with higher recognition accuracies for the possible objects $(F(1,13)=36.33, P<0.0001)$, but no effect of presentation $(F(3,39)=1.60, P=0.20)$ and no interaction between object type and presentation $(F(3,39)=2.62$, $P=0.06$ ). For completeness, we also computed the signal detection measures discriminability, $d^{\prime}$, and bias, $C_{l n}$. There was no effect of presentation on either $d^{\prime}(F(3,39)=0.82$, $P=0.49)$ or $C_{\ln }(F(3,39)=1.57, P=0.21$ ). (All corrected according to Huyn-Feldt epsilon criterion.)

To check for the possibility of a mere strengthening of the stimulus-response association (Dobbins et al., 2004; Schacter et al., 2004) at the expense of a true object decision, we analyzed the accuracies for subsequent responses, given that prior responses to an object were incorrect. Specifically, we denote with $P\left(I_{N} \mid I_{N}-1\right)$ and $P\left(C_{N} \mid I_{N}-1\right)$ the probability that the Nth object decision was incorrect or correct, respectively, given that the previous $(N-1)$ th decision regarding the same object was incorrect. Obviously, $P\left(I_{N} \mid I_{N-1}\right)+P\left(C_{N} \mid I_{N-1}\right)=1$. We found no indication that there is a tendency to persist with an incorrect object decision: $P\left(I_{2} \mid I I_{1}\right)=0.50, P\left(I_{3} \mid I_{2}\right)=0.46$, $P\left(I_{4} \mid I_{3}\right)=0.38$. Subjects appear to engage in the object decision task; they are not just relying on a strengthening of stimulusresponse association.

\section{2. fMRI data}

For the purposes of the MR image analysis, our experiment contained the main design parameter of presentation order (1st, 2nd, and 3rd) for possible objects, and data from all three presentations were submitted to the covariance analysis to identify an activation pattern that systematically decreased its expression across presentations for as many subjects as possible. We found such a pattern as a linear combination of the first two principal components whose expression decreased across presentations for the majority of subjects (permutation test: $r m-F=8.02, P<0.01$ ). This pattern accounted for $16 \%$ of the variance in the data. In addition, the decrease in pattern expression from presentation 1 to 2 was 
predictive of the corresponding reaction time decrease $\left(R^{2}=0.67, P<0.0005\right)$ (Fig. 3).

A set of occipital, parietal, and temporal brain regions decreased their activation across presentations, including bilateral middle occipital gyrus, left precuneus, right supramarginal gyrus, as well as some frontal and thalamic areas, such as right inferior frontal gyrus, left cingulate gyrus, and right thalamus. Apart from this set of areas showing repetition suppression, there is also a set of areas showing concomitant repetition enhancement (see Table 3 and Fig. 4).

When we conducted a similar analysis on the data from the first three presentations for the I-objects and found a pattern whose subject expression decreases across the first 3 presentations from the first 3 principal components (permutation test: $r m-F=5.46, P<0.001$; graph not shown). This pattern accounted for $10 \%$ of the variance in the data. Unfortunately, the bootstrap resampling process did not yield any sufficiently robust regions that surpassed a threshold of $|Z|>3.09$. Contrary to the P-related pattern, the subject expression of the I-related pattern could not account for any reaction time priming behavior; the decrease in expression from presentation 1 to 2 did not yield a significant correlation with the corresponding reaction time decrease $\left(R^{2}=0.10, P=0.27\right)$.

Whether both object-specific activation patterns underlying repetition suppression/enhancement are similar is difficult to answer directly. Both patterns consist of voxel weights that could be subjected to a correlation analysis, but it is not clear how to correctly test the null-hypothesis that both patterns are unrelated to each other. Linear regression approaches or permutation tests that permute voxel locations would produce highly significant $P$ values that are probably not conservative enough, since the spatial correlation induced by the smoothing process is not taken into account adequately, resulting in an artificially high number of degrees of freedom.
The issue of similarity of the covariance patterns can be addressed through forward application of each pattern to the data of the other object type that did not give rise to the pattern in the first place. We first investigated whether the activation pattern underlying repetition effects and RT priming in Pobjects could be forward applied successfully to the I-objects. The rationale was the following: it is conceivable that the $P$ related activation pattern is equally operational in both object types, accounting for both repetition effects and RT priming, but in the case of I-objects, its activation might be superseded by large variance in the data contributed by other effects, making a clean identification in an analysis of the data from presentation of I-objects impossible. If so, subjects' expression of the activation pattern should still show a repetition effect and a correlation with RT priming. This was, however, not the case: neither did the subjects' expression correlate significantly with RT priming, nor was there a repetition effect (permutation test: $r m-F=0.95, P=0.43$ ). The reverse application of the I-specific pattern to the data from presentations of the Pobjects also did not yield any repetition effects $(r m-F=0.13$, $P=0.86)$. The neural underpinnings of both object types' repetition effects are therefore too specific to permit generalization to the other object type.

\section{Discussion}

In a repeated object decision task, we found a neural activation pattern that showed repetition effects across the first three presentations of three-dimensionally possible objects and additionally accounted for reaction time priming. An activation pattern manifesting similar repetition effects could be found for the impossible objects but did not account for any reaction time priming. Although subjects' reaction time priming behavior was similar for both types of objects, it
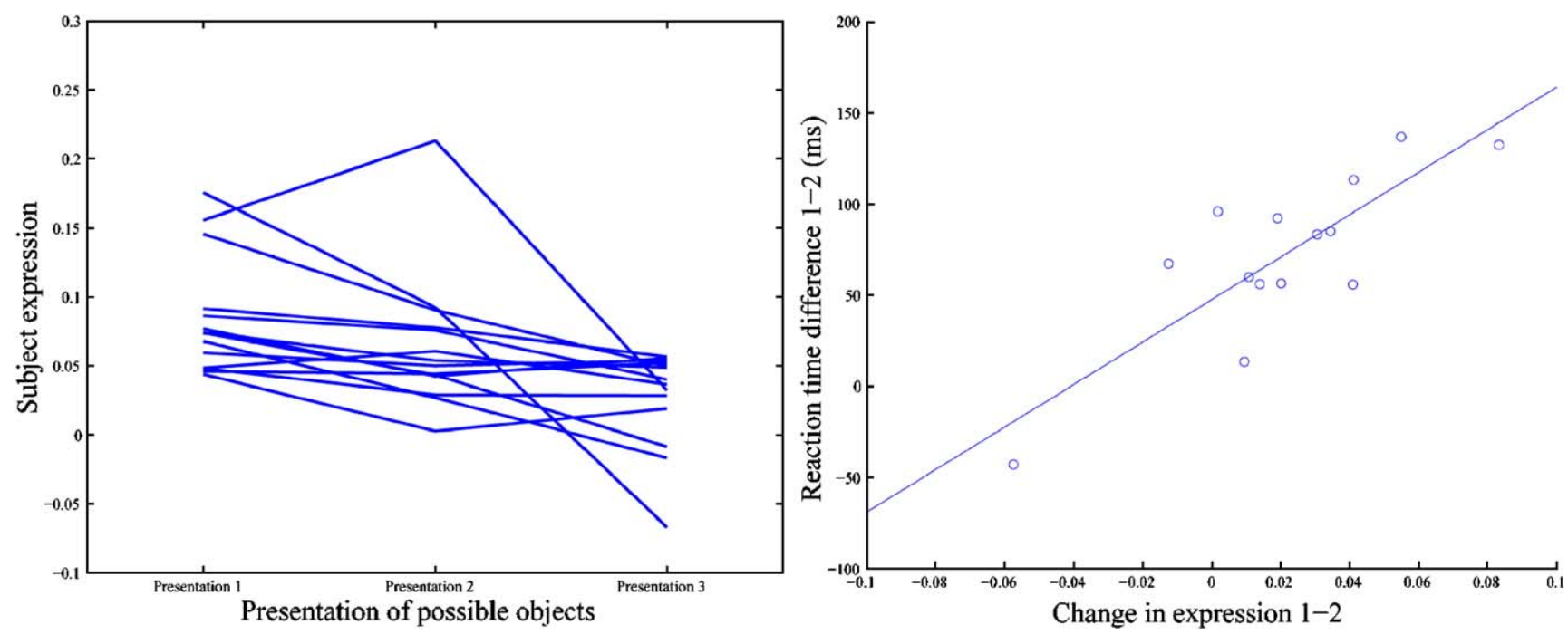

Fig. 3 - Relationship between subjects' expression of the activation pattern and experimental and behavioral variables. Left: subject expression of activation plotted versus presentation number. Most subjects decrease their expression of the activation pattern monotonically across presentations $(\mathrm{rm}-\mathrm{F}=\mathbf{8 . 0 1}, \mathrm{P}<0.001)$. Right: reaction time difference between $1 \mathrm{st}$ and $2 \mathrm{nd}$ presentations plotted versus the corresponding difference in the expression of the activation pattern. The amount of decrease in pattern expression could predict the magnitude of the $R T$ priming effect $\left(R^{2}=0.67, P<0.0005\right)$. 
Table 3 - Nearest gray matter voxel locations of positive and negative weights $(|Z|>3.09)$ in the neural activation pattern underlying repetition effects in P-objects: listed under 'positive' are MNI coordinates of gray matter structures that are increasing their activation with repeating stimulus presentation; 'negative' denotes areas that are decreasing their activation with repeating stimulus presentation

\begin{tabular}{lrrll} 
X & Y & Z & \multicolumn{1}{c}{ Structure } & Brodmann label \\
\hline \multicolumn{2}{l}{ Positive } & & & \\
28 & -84 & 16 & Middle occipital gyrus & Brodmann area 19 \\
-24 & -82 & 34 & Precuneus & Brodmann area 19 \\
-12 & -84 & 38 & Precuneus & Brodmann area 19 \\
-24 & -73 & 26 & Precuneus & Brodmann area 31 \\
42 & -54 & -17 & Fusiform gyrus & Brodmann area 37 \\
34 & -56 & 51 & Superior parietal lobule & Brodmann area 7 \\
24 & -58 & 56 & Precuneus & Brodmann area 7 \\
16 & -28 & -2 & Thalamus & * \\
-26 & -88 & 16 & Middle occipital gyrus & Brodmann area 19 \\
51 & 10 & 22 & Inferior frontal gyrus & Brodmann area 44 \\
28 & -38 & -11 & Parahippocampal gyrus & Brodmann area 36 \\
-42 & -42 & 38 & Supramarginal gyrus & Brodmann area 40 \\
& & & & \\
Negative & & & \\
44 & -70 & 42 & Inferior parietal lobule & Brodmann area 39 \\
-18 & -38 & -11 & Parahippocampal gyrus & Brodmann area 36 \\
-8 & -58 & 46 & Precuneus & Brodmann area 7 \\
-12 & -52 & 52 & Precuneus & Brodmann area 7 \\
-38 & -16 & 60 & Precentral gyrus & Brodmann area 4 \\
-40 & -8 & 56 & Precentral gyrus & Brodmann area 6 \\
-40 & -14 & 38 & Precentral gyrus & Brodmann area 6 \\
-44 & -72 & 13 & Middle temporal gyrus & Brodmann area 39 \\
-48 & -72 & 36 & Angular gyrus & Brodmann area 39 \\
-54 & -36 & 26 & Inferior parietal lobule & Brodmann area 40 \\
-20 & -54 & 65 & Postcentral gyrus & Brodmann area 7 \\
-46 & -56 & 30 & Superior temporal gyrus & Brodmann area 39 \\
6 & -44 & 50 & Precuneus & Brodmann area 7 \\
-16 & 0 & 26 & Caudate & Caudate body \\
\hline
\end{tabular}

Localization with Talairach Demon available from http://ric. uthscsa.edu/projects/talairachdaemon.html.

seems that a neural repetition effect can only account for this priming behavior in the case of the P-objects.

These findings might point to fundamental differences in the way possible and impossible objects are stored and processed in the brain. When the pattern underlying the repetition effects in each object type was forward applied to the data from the other object type, no systematic change in expression across presentations could be obtained, emphasizing the object type specificity of each pattern.

Although the impossible objects, while showing overall slower reaction times than possible objects, manifested reaction time priming from first to second presentation that was similar to the possible objects, the neural underpinnings of this priming effect are apparently quite different between the two object types. A structural descriptive system (SDS) was postulated some time ago (Schacter and Cooper, 1993; Schacter et al., 1990, 1991) and aids the encoding of, and access to, implicit memory of structurally possible information is consistent with the brain regions underlying the repetition and priming effects for possible objects. However, in the cited studies, there was no priming for impossible objects, offering prima facie evidence that the SDS was simply not operational for impossible objects. The results reported here complicate this picture: since priming of similar magnitude was observed for both impossible and possible objects, one has to ask what neural underpinnings give rise to priming of impossible objects, if not the SDS? It is conceivable that underlying the reaction time priming for impossible objects, there is focal activation that does not show a consistent repetition effect and does not contribute a lot of variance in the data. Our multivariate analysis might then be likely to miss such a pattern of activation. However, such an explanation seems unlikely: impossible objects are not encountered in everyday life and thus do not merit a high degree functional specialization of selected brain regions in their response. It seems more likely that a coalition of frontal and parietal brain regions involved in memory and spatial attention might be recruited for the classification of impossible objects in a manner that is highly variable from individual to individual. The discovery of such effects necessitates more specialized multivariate analysis and the utilization of subject-specific information (reaction time, recognition accuracy) in tailored design matrices.

Our paper did not explore the possibility that the obtained results might in part be due to differences in consistency for local and global processing. Possible objects are possible at both the local and global levels of analysis, while impossible objects are globally impossible, but locally possible. It should be noted that at the local level, there is generally no such thing as structural impossibility. Impossibility arises in the structural incompatibility across regions or parts. Hence, structural impossibility is really only a global object attribute, and differences could be attributable to general processing considerations and not specific to object structure. In the current study, the instructions given to the subjects strongly emphasized global processing, and both structural possibility and impossibility were strongly defined as global properties. We thus tried to minimize the global/local-confound but cannot entirely rule it out.

We conclude with the general observation that the neural mechanisms of rapid perceptual learning involve a variety of different brain areas that show both response suppression and enhancement in the case of possible objects. The neural correlates of perceptual learning are therefore unlikely to be reducible to exclusive neural response suppression of lateral occipital regions (Dolan et al., 1997; Henson and Rugg, 2003) but also entail the involvement of occipital, frontal, and parietal areas for processing stimulus attributes, spatial attention, and feature binding, as well as those involved in visual imagery. Neither is it clear that perceptual learning necessarily always involves neural repetition effects, as shown in this report for the class of structurally impossible objects. The I-objects' neural repetition effects could not account for the observed reaction time priming in this study. This indicates either (1) that the neural underpinnings of the behavioral priming effect cannot be described as a repetition effect of similarly signed changes in activation in response to repeated stimulation on a subject-by-subject basis, or (2) that there is a neural repetition effect underlying behavioral priming for I-objects, but its variance contribution is too small to be captured by our analysis technique. For completeness, we should mention that in addition to monotonically changing neural correlates, 
A

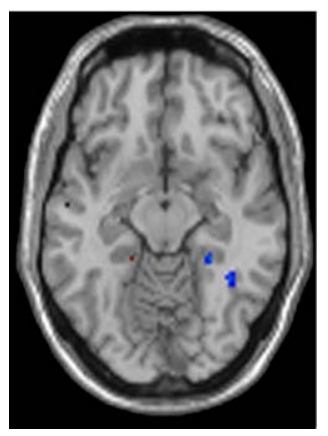

B

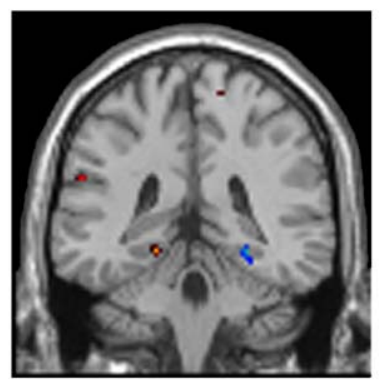

C

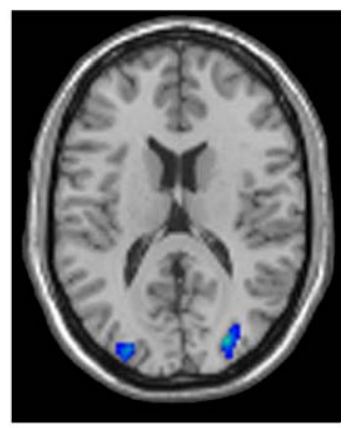

Fig. 4 - Plot of axial and coronal slices (MRIcro, available from www.mricro.com) in the P-related activation pattern, showing selected voxel locations that showed stable regional weights as ascertained by the bootstrap resampling procedure $(|Z|>3.09$, $P<0.001)$. Cold colors denote areas that are showing response suppression across presentations; hot colors denote areas that are showing response enhancement. (A) Axial slice with MNI coordinate $z=-10 \mathrm{~mm}$; repetition suppression of the hemodynamic response can be discerned at the location of right fusiform and parahippocampal gyrus, whereas repetition enhancement is found for the left parahippocampal gyrus. (B) Coronal slice with MNI coordinate $y=-38$, illustrating the response sign-switch for the parahippocampal gyri, depending on laterality; additionally, one can clearly discern repetition enhancement in the left inferior parietal lobule (BA 40). (C) Axial slice with MNI coordinate $z=+16 \mathrm{~mm}$; extended foci of repetition suppression can be seen in bilateral occipital gyrus (BA 19).

the first possibility also exists for the P-objects, i.e., activation patterns that do not show a monotonic increase in expression on a subject-by-subject basis yet play some role in priming. In either case, there is a clear need for the reliable elucidation of subject and task effects that determine the interaction of stimulus material, on the one hand, and the link between neural repetition effects and perceptual learning, on the other hand.

\section{Experimental procedures}

Fourteen subjects (age $=22.8 \pm 3.8$ years; range $16-29$ years) participated in an event-related functional magnetic resonance imaging (efMRI) study of a continuous object decision task. All subjects were right-handed and carefully screened to ensure that they had no history of medical, psychiatric, neurological, or sleep disorders. Informed consent, as approved by the Internal Review Board of the College of Physicians and Surgeons of Columbia University, was obtained prior to study participation and after the nature and risks of the study were explained. Subjects were paid for their participation in the study.

\subsection{Possible/Impossible object decision repetition priming task \\ 4.1.1. Stimuli}

The stimuli consisted of images of 39 three-dimensionally possible and 39 impossible line-drawn objects. The 78 test stimuli were selected from a larger set of 278 candidate items (154 Possible; 124 Impossible) and assigned to one of three balanced object sets on the basis of earlier behavioral pilot experiments. An additional 10 stimuli were selected as buffer items used at the beginning of blocks and as practice items.

\subsubsection{Procedure}

The possible/impossible object decision repetition priming task consisted of three test blocks, each with a distinct set of 26 stimuli (13 possible; 13 impossible). The assignment of object sets to test block was counterbalanced across subjects such that each object set occurred equally often in the first, second, and third test blocks. Subjects were instructed to indicate whether each stimulus represented a three-dimensionally possible, or impossible object, acting as quickly and as accurately as possible. Each of the test items was viewed four times in a specific repetition sequence. However, given that only 13 objects of each type were presented in a block, each block had 5 of one sequence pattern and four of each of the others. Each block consisted of 52 possible (13 objects $\times 4$ presentations) trials and 52 impossible trials. An additional 52 blank trials were added to allow for the tight interleaving of the of the object sequences within a block in order to maintain the required object spacing and to serve as positive controls. Participants were informed that blank trials would occur, and that no response should be made during these events.

All trials were exactly $3000 \mathrm{~m}$ in duration and were automatically initiated by the testing computer. Each trial began with a 500-m ITI, followed by a fixation cue ("+") for 250 ms. Fifty milliseconds after fixation offset, the stimulus then appeared for $1000 \mathrm{~ms}$. Responses with a differential button press ("left hand" for impossible; "right hand" for possible) were registered from stimulus onset until the end of the trial using an fMRI compatible LUMItouch response system. In blank trials, the fixation cue was presented, but no stimulus followed. Stimulus presentations were automatically synched to the video retrace signal. Reaction time measurement began with the actual stimulus onset referenced by the video retrace signal. All timing was facilitated by use of the external PsyScope Button Box, which interfaces directly with the PsyScope software and host computer and also provides timing accurate to $1 \mathrm{~ms}$. The PsyScope Button Box also provides the connection to the LUMItouch response system. An additional four buffer trials were added to the beginning of every block, making the blocks exactly $8 \mathrm{~min}$ in duration. Immediately prior to the onset of the experiment, possible and impossible were defined by the experimenter, and several examples of each type of object were shown. The characteristic line features that distinguished the sample objects as three-dimensionally impossible were pointed out to the participants. If they expressed uncertainty as to these definitions, the distinctions were reviewed. Participants were not told in advance that the procedure itself was a test of memory. Five practice trials were then presented after which participants were asked if they understood the task. 


\section{2. $\quad$ fMRI acquisition and processing}

Functional images were acquired using a 1.5- $\mathrm{T}$ magnetic resonance scanner (Philips). A gradient echo EPI sequence (TE $=50 \mathrm{~ms}$; $\mathrm{TR}=3 \mathrm{sec}$; flip angle $=90^{\circ}$ ) and a standard quadrature head coil was used to acquire $\mathrm{T} 2{ }^{*}$-weighted images with an in-plane resolution of $3.124 \mathrm{~mm} \times 3.124 \mathrm{~mm}\left(64 \times 64\right.$ matrix; $20-\mathrm{cm}^{2}$ field of view). Based on $\mathrm{T}_{1}$ "scout" images, $8 \mathrm{~mm}$ transaxial slices (15-17) were acquired. Following the fMRI runs, a high (in-plane) resolution $\mathrm{T}_{2}$ image at the same slice locations used in the fMRI run was acquired using a fast spin echo sequence (TE $=100 \mathrm{~ms} ; \mathrm{TR}=3 \mathrm{~s} ; 256 \times 256$ matrix; 20$\mathrm{cm}^{2}$ field of view). Task administration and data collection were controlled by a computer running PsyScope 1.1 experiment development package (Cohen, MacWhinney, Flatt and Provost, 1993), and electronically synchronized with the MR scanner. Task stimuli were back projected onto a screen located at the foot of the MRI bed using an LCD projector. Subjects viewed the screen via a mirror system located in the head coil. Task responses were made on a LUMItouch MRI compatible response system, and behavioral response data were recorded on the task computer.

All image processing and analysis were done using the SPM99 program (Wellcome Department of Cognitive Neurology) and other code written in Matlab 5.3 (Mathworks, Natick MA). fMRI time series were corrected for order of slice acquisition. All functional volumes for a given subject were realigned to the first volume from the first run of each study. The $\mathrm{T}_{2}$ anatomical image was then coregistered to the first functional volume, using the mutual information co-registration algorithm implemented in SPM99. This co-registered structural image was then used in determining nonlinear spatial normalization $(7 \times 8 \times 7$ nonlinear basis functions) parameters for a transformation into a Talairach standard space defined by the Montreal Neurological Institute template brain applied with SPM99. These normalization parameters were then applied to the functional data (using SINCinterpolation to reslice the images to $2 \mathrm{~mm} \times 2 \mathrm{~mm} \times 2 \mathrm{~mm}$ ).

\subsection{Data analysis}

The fMRI responses to the all components of the task, in each experimental condition and in each block, were fit to separate sets of predictor variables (Zarahn, 2000). The predictor variables that were ultimately used in the first-level model estimation were obtained in the following way: a constant intercept was chosen. Predictor variables had a nonzero value at every point in the time series where a particular condition was met and a zero value at every other point. For example, one predictor had a value of one during all possible objects during the first block.

The set was convolved with a canonical hemodynamic response waveform (a sum of two gamma functions, as specified in the SPM99 program (Friston et al., 1998)) whose beginnings were marked by the appropriate onset vector for each epoch, repetition of presentation, and block. The resulting time series vectors were used in the design matrix for the within-subject model estimation. The final matrix had 4-by-4 block-diagonal form. The number of rows was the total number of volumes denoting the complete fMRI time series across the scanning session. The number of columns was the total number of predictors $(=4 \times(2+1)=12)$.

The high-pass filtered (cutoff of $12.8 \mathrm{MHz}$ ) fMRI time series at each voxel were regressed onto these predictor variables. A first order autoregressive autocorrelation model was fit to the residuals to make statistical inference more robust to the intrinsic temporal autocorrelation structure (Friston et al., 2000).

At every voxel in the image, contrasts assessed the amplitudes (normalized regression coefficients) of the components of the event-related responses that matched the canonical hemodynamic response waveform for the whole scanning session. A typical contrast used in our analysis for instance would be "activity while viewing possible figures for the first time collapsed across experimental blocks versus activity in the ITI blank period". This method of time series modeling and contrast estimation at each voxel reduces the number of images to one per subject per condition. To account for gain differences between fMRI sessions, activation values were normalized by their voxel averages. The resulting parametric maps of normalized regression weights were smoothed using an isotropic Gaussian kernel (FWHM $=8 \mathrm{~mm}$ ) and used for the subsequent population-level multivariate analysis. Six parametric maps were used: one for each of 3 presentations (1/2/3) for each of 2 object types (possible/impossible).

\subsection{Multivariate analysis}

Ordinal trend canonical variates analysis (OrT CVA) (Habeck et al., 2002, 2004, 2005) was performed on the data. This analysis is similar to other regional covariance analyses techniques, notably partial least squares, to the extent that it applies principal components analysis (PCA) to the data matrix that is transformed using a matrix representing the experimental design (McIntosh et al., 1996; Worsley et al., 1997). The design matrix of OrT CVA has been optimized to increase the variance contribution (and subsequent detectability in the PCA) of effects that show consistency in their behavior across conditions and subjects (Habeck et al., 2005). A subsequent linear regression is employed to identify a covariance pattern in the MR signal with decreasing mean subject expression across the first three of the four presentations from a linear combination involving a small set of principal components. The property of a consistent monotonic within-subject change of pattern expression across task conditions (beyond mere mean change) is called an "ordinal trend". The combination of a PCA after data transformation with our specialized design matrix and a subsequent linear regression is very sensitive to the presence of ordinal trends in the data (Habeck et al., 2005).

At present, OrT CVA is only equipped to utilize the first three presentations for the derivation of the covariance pattern showing a repetition effect. However, any covariance pattern can always be applied prospectively to any data set, even if this data set was not used in the original derivation of the pattern.

Individual subject's expression of this activation pattern is quantified with the subject-scaling factor (SSF). The SSF is obtained by the operation of an inner product (=covariance across brain regions) between the covariance pattern in question and a subject's task scan. It quantifies to what extent a subject expresses the activation pattern in a task scan with a single number, which can be used for further analysis. The activation attributable to the covariance pattern can then be written as

\section{Activation $=\mathrm{SSF} \times$ covariancepattern}

Referring back to Fig. 2 for instance, we observe that all three regions of interest have a loading of 1 , i.e., the covariance pattern can be denoted as $[1 ; 1 ; 1]$. A level of expression of 1 means that the activation attributable to the covariance pattern is $[1 ; 1 ; 1]$; a level of expression of 2 implies an activation pattern [2; 2; 2]. Decreasing the level of expression from 4 to 2 to 1 across repetitions of the stimulus for instance would imply the following chain of activation patterns $[4 ; 4 ; 4] \rightarrow[2 ; 2 ; 2] \rightarrow[1 ; 1 ; 1]$. To avoid any confusion here, we stress that the regional weights for this mock example were chosen as equal, but in general, they will display different magnitude and sign for different regions.

Ordinal trends can be quantified with the repeated measures F statistic ("rm-F"), constructed from the SSF values that were obtained by projecting the covariance pattern in question onto every subject's task scans. For instance, if there are 15 subjects and 3 conditions, there will be 45 SSF values from which the rm$F$ statistic can be computed. The set of principal components that was used to construct the covariance pattern needs to be taken into consideration here too, since using more principal components to produce a linear combination pattern to fit a particular expression behavior will result in larger $P$ values. For 
equal values of the $\mathrm{rm}-\mathrm{F}$ statistic for instance, a covariance pattern that was produced from principal components 1 and 2 is therefore less likely to have been obtained by chance than a pattern that was made up from principal components 1-5.

A common challenge for multivariate analysts is the decision how many principal components to include in any subsequent analyses (i.e., see, Burnham and Anderson, 2002; Hansen et al., 1999). The decision rule we adopted was the following: we started with the principal component of the largest variance contribution and successively added the lower-variance components, i.e., the succession was PC1, PC1-2, PC1-3, and PC1-4, etc. Each time, we performed a multiple linear regression using the SSFs of all PCs in the set as independent variables to construct a pattern that showed a maximal mean change across repetitions. Of those, we chose the linear combination pattern whose model fit showed the lowest value of the Akaike information criterion (AIC) (Burnham and Anderson, 2002). Briefly, the AIC takes into account the maximum likelihood value achieved in the regression and pits it against the number of parameters used, indicating the best bias variance trade-off between under- and overfitting the data. It should not be misinterpreted as a statistical test of significance of the ordinal trend, which follows in the next paragraph.

For the significance test of the selected linear combination pattern, a random permutation test generates the $P$ level for the $\mathrm{rm}-\mathrm{F}$ statistic actually observed for the activation pattern in our experimental sample. For these simulations, we destroyed the condition assignment and analyzed the within-subjects resampled data 10,000 times, employing exactly the same set of principal components that was recruited in the linear combination to produce the point estimate of the pattern in our subject sample.

An ordinal trend lends additional credence to the claim that an activation pattern was obtained through the experimental design manipulation, rather than a mean change across conditions that might have come about purely as a result of overly influential subject outliers. This added across-subjects consistency improves the sensitivity of the method without any sacrifice of specificity.

Activation patterns resulting from multivariate analysis assign different weights to all voxels included in the analysis, depending on the salience of their covariance contribution. Positive voxel weights indicate a positive correlation between the subject expression value and the associated regional activation, whereas negative weights indicate a negative correlation. This means that as the expression of a pattern increases, activation in the positively weighted regions increases as well, whereas activation in the negatively weighted regions decreases. Whether a voxel weight is reliably different from zero is assessed by a bootstrap estimation procedure (Efron and Tibshirani, 1994). Denoting the point estimate of a voxel weight as $\mathrm{w}$ and the standard deviation resulting from the bootstrap resampling procedure as $s_{\mathrm{w}}$, we can compute a $\mathrm{z}$ score according to $\mathrm{z}=\mathrm{w} / \mathrm{s}_{\mathrm{w}}$. Sufficiently small variability of a voxel weight around its point estimate value in the resampling processes results in $z$ value of large magnitude and indicates a reliable contribution to the covariance pattern. As the threshold criterion we chose $|z|>3.09$; under the assumptions of a standard normal distribution, i.e., $\mathrm{z} \sim \mathrm{N}(0,1)$, this corresponds to a one-tailed probability of 0.001 .

Once an activation pattern was identified that systematically increases in expression across presentation order, we examined the correlation between individual change in network expression from presentations 1 to 2 (SSF(1)-SSF(2)) and the corresponding change in their reaction times.

\section{Acknowledgment}

This work was supported by NIH/NIA RO1 AG16714.

\section{RE F EREN C ES}

Brown, M.W., Xiang, J.Z., 1998. Prog. Neurobiol. 55, 149-189. Burnham, K.P., Anderson, D.R., 2002. Model Selection and Multimodel Inference. Springer Verlag, New York.

Desimone, R., 1996. Proc. Natl. Acad. Sci. U. S. A. 93, 13494-13499.

Dobbins, I.G., Schnyer, D.M., Verfaellie, M., Schacter, D.L., 2004. Nature 428, 316-319.

Dolan, R.J., Fink, G.R., Rolls, E., Booth, M., Holmes, A., Frackowiak, R.S., Friston, K.J., 1997. Nature 389, 596-599.

Efron, B., Tibshirani, R.J., 1994. An Introduction to the Bootstrap. CRC Press, LLC, New York.

Eger, E., Henson, R.N., Driver, J., Dolan, R.J., 2004. J. Neurophysiol. 1241-1247.

Friston, K.J., Fletcher, P., Josephs, O., Holmes, A., Rugg, M.D., Turner, R., 1998. NeuroImage 7, 30-40.

Friston, K.J., Josephs, O., Zarahn, E., Holmes, A.P., Rouquette, S., Poline, J., 2000. NeuroImage 12, 196-208.

Grill-Spector, K., Kushnir, T., Edelman, S., Avidan, G., Itzchak, Y., Malach, R., 1999. Neuron 24, 187-203.

Habeck, C.G., Stern, Y., Posner, H.B., Moeller, J.R., 2002. SFN Abstracts.

Habeck, C., Rakitin, B.C., Moeller, J., Scarmeas, N., Zarahn, E., Brown, T., Stern, Y., 2004. Brain Res. Cogn. Brain Res. 18, 306-321.

Habeck, C., Krakauer, J.W., Ghez, C., Sackeim, H.A., Eidelberg, D., Stern, Y., Moeller, J.R., 2005. Neural Comput. 17, 1602-1645.

Hansen, L.K., Larsen, J., Nielsen, F.A., Strother, S.C., Rostrup, E., Savoy, R., Lange, N., Sidtis, J., Svarer, C., Paulson, O.B., 1999. NeuroImage 9, 534-544.

Henson, R.N., Rugg, M.D., 2003. Neuropsychologia 41, 263-270.

Henson, R.N., Shallice, T., Gorno-Tempini, M.L., Dolan, R.J., 2002. Cereb. Cortex 12, 178-186.

Kourtzi, Z., Kanwisher, N., 2000. J. Neurosci. 20, 3310-3318.

Kourtzi, Z., Kanwisher, N., 2001. Science 293, 1506-1509.

Logothetis, N.K., Pauls, J., Augath, M., Trinath, T., Oeltermann, A., 2001. Nature 412, 150-157.

McIntosh, A.R., Bookstein, F.L., Haxby, J.V., Grady, C.L., 1996. NeuroImage 3, 143-157.

Schacter, D.L., Buckner, R.L., 1998. Neuron 20, 185-195.

Schacter, D.L., Cooper, L.A., 1993. J. Exp. Psychol., Learn Mem. Cogn. 19, 995-1009.

Schacter, D.L., Cooper, L.A., 1995. J. Exp. Psychol., Learn Mem. Cogn. 21, 768-776.

Schacter, D.L., Cooper, L.A., Delaney, S.M., 1990. J. Exp. Psychol. Gen. 119, 5-24.

Schacter, D.L., Cooper, L.A., Delaney, S.M., Peterson, M.A., Tharan, M., 1991. J. Exp. Psychol., Learn Mem. Cogn. 17, 3-19.

Schacter, D.L., Reiman, E., Uecker, A., Polster, M.R., Yun, L.S., Cooper, L.A., 1995. Nature 376, 587-590.

Schacter, D.L., Dobbins, I.G., Schnyer, D.M., 2004. Nat. Rev., Neurosci. 5, 853-862.

Wiggs, C.L., Martin, A., 1998. Curr. Opin. Neurobiol. 8, 227-233.

Worsley, K.J., Poline, J.B., Friston, K.J., Evans, A.C., 1997.

NeuroImage 6, 305-319.

Zarahn, E., 2000. NeuroImage 11, 783-796. 